\title{
A necessary and sufficient condition for exponentially bounded existence and uniqueness
}

\section{David Lowell Lovelady}

A condition which was previously found to be sufficient for global existence and uniqueness of solutions of an ordinary differential equation is shown herein to be necessary, if it is also required that solutions are exponentially bounded.

Let $Y$ be a Banach space with norm || , let $A$ be a continuous function from $[0, \infty) \times Y$ to $Y$, and let $\alpha$ be a continuous real-valued function on $[0, \infty)$. In [2] this author and Martin showed that (I) implies (II).

(I) If $(t, x, y)$ is in $[0, \infty) \times y \times Y$ and $c$ is a positive number then

$$
|x-y-c[A(t, x)-A(t, y)]| \geq[1-c \alpha(t)]|x-y| \text {. }
$$

(II) If $(a, x)$ is in $[0, \infty) \times y$ there is exactly one continuously differentiable function $u$ from $[a, \infty)$ to $y$ such that $u(a)=x$ and

(1)

$$
u^{\prime}(t)=A(t, u(t))
$$

whenever $t$ is in $[a, \infty)$. Furthermore, if $v$ also solves

(1) and $v(a)=y$ (where $y$ is in $Y$ ) then

$$
|u(t)-v(t)| \leq|x-y| \exp \left[\int_{a}^{t} \alpha(s) d s\right]
$$




$$
\text { whenever } t \text { is in }[a, \infty) \text {. }
$$

In the present note we shall show that (II) implies (I), and hence we have the following theorem.

THEOREM. Statements (I) and (II) are equivalent.

From this point forward we shall assume that (II) is true. Let $m_{-}$ be given on $Y \times Y$ by

$$
m_{-}[x, y]=\lim _{\delta \rightarrow 0-}(1 / \delta)(|x+\delta y|-|x|),
$$

and note that if $(x, y)$ is in $Y \times y$ then

$$
m_{-}[x, y]=\sup \{(1 / \delta)(|x+\delta y|-|x|): \delta \text { is a negative number }\}
$$

(compare $[1$, p. 3]). Hence $(I)$ is equivalent to requiring that

$$
m_{-}[x-y, A(t, x)-A(t, y)] \leq \alpha(t)|x-y|
$$

whenever $(t, x, y)$ is in $[0, \infty) \times y \times y$, and this is what we shall show. Let $m_{+}$be given on $Y \times Y$ by

$$
m_{+}[x, y]=\lim _{\delta \rightarrow 0^{+}}(1 / \delta)(|x+\delta y|-|x|),
$$

and recall that $m_{-}[x, y] \leq m_{+}[x, y]$ whenever $(x, y)$ is in $y \times y$ (compare [1, p. 3]). Also, if $f$ is a function from a subset of $[0, \infty$ ) to $y$, if $c$ is in the domain of $f$, if $f_{+}^{\prime}(c)$ (the right derivative of $f$ at $c$ ) exists, and if $p$ is given on the domain of $f$ by $p(t)=|f(t)|$, then $p_{+}^{\prime}(c)$ exists and $p_{+}^{\prime}(c)=m_{+}\left[f(c), f_{+}^{\prime}(c)\right]$ (see $[1$, p. 3]).

Now let $a, x, y, u$, and $v$ be as in (II). Let $p$ be given on $[a, \infty)$ by $p(t)=|u(t)-v(t)|$. Now

$$
\begin{aligned}
m_{-}[x-y, A(a, x)-A(a, y)] & \leq m_{+}[x-y, A(a, x)-A(a, y)] \\
& =p_{+}^{\prime}(a) \\
& =\lim _{\delta \rightarrow 0+}(1 / \delta)(p(a+\delta)-|x-y|) \\
& \leq|x-y| \lim _{\delta \rightarrow 0+}(1 / \delta)\left\{\exp \left[\int_{a}^{a+\delta} \alpha(s) d s\right]-1\right\} \\
& =|x-y| \alpha(a),
\end{aligned}
$$


and the proof is complete.

\section{References}

[1] W.A. Coppel, Stability and asymptotic behavior of differential equations (D.C. Heath, Boston, 1965).

[2] David Lowell Lovelady and Robert H. Martin, Jr, "A globel existence theorem for a noneutonomous differential equation in a Banach space", Proc. Amer. Math. Soc. 35 (1972), 445-449.

Department of Mathematics,

Florida State University,

Tal lahassee,

Florida,

USA. 